\title{
COMBINATORIAL MULTINOMIAL MATRICES AND MULTINOMIAL STIRLING NUMBERS
}

\author{
DANIEL S. MOAK
}

\author{
(Communicated by Thomas H. Brylawski)
}

Abstract. Fred C. Barnett and James R. Weaver considered the stochastic matrix

$$
\left[\left(\begin{array}{l}
n \\
j
\end{array}\right)\left(\frac{n-i}{n}\right)^{n-j}\left(\frac{i}{n}\right)^{j}\right]_{i, j=0, \ldots, n}
$$

when modeling the spread of a viral infection through a population, where the virus has two forms. This can be generalized to viruses with $q$ forms using the matrix

(2)

$$
\left[\left(\begin{array}{c}
n \\
\beta_{1}, \beta_{2}, \ldots, \beta_{q}
\end{array}\right)\left(\frac{\alpha_{1}}{n}\right)^{\beta_{1}}\left(\frac{\alpha_{2}}{n}\right)^{\beta_{2}} \cdots\left(\frac{\alpha_{q}}{n}\right)^{\beta_{q}}\right]_{\alpha_{1}+\alpha_{2}+\cdots+\alpha_{q}=n, \beta_{1}+\beta_{2}+\cdots+\beta_{q}=n}
$$

These matrices also appear in a different context when Konrad J. Heuvers, et al, studied the characterization of the permanent function by the Cauchy-Binet formula. In this paper, the eigenvalues and inverse of the matrix (2) are given and the existence of a basis of right eigenvectors is established. found.

In the process the inverse of a generalized multinomial coefficient matrix is

\section{INTRODUCTION}

Consider a virus that exists in $q$ forms, $A_{1}, A_{2}, \ldots, A_{q}$, and assume that all $q$ of these forms reproduce at the same high rate. Assume also that $n$ particles of a virus are transmitted by a vector which selects these $n$ particles randomly from an effectively infinite number of virus particles in the infected host. Some time after the vector infects the new host, the new host will have the same proportion of the $q$ forms of the virus as he received from the vector. Consequently if a host receives $\alpha_{1}$ particles of type $A_{1}, \alpha_{2}$ particles of type $A_{2}$, etc., then the probability that the next host will have $\beta_{1}$ particles of type

Received by the editors September 9, 1987 and, in revised form, October 31, 1988. Presented at the 93rd annual meeting, American Mathematical Society, San Antonio, Texas, January 1987.

1980 Mathematics Subject Classification (1985 Revision). Primary 15A15, 39B60; Secondary $05 \mathrm{~A} 10$ 
$A_{1}, \beta_{2}$ particles of type $A_{2}$, etc. will be

$$
\begin{aligned}
D_{\alpha, \beta}=\left(\begin{array}{c}
n \\
\beta_{1}, \beta_{2}, \ldots, \beta_{q}
\end{array}\right)\left(\frac{\alpha_{1}}{n}\right)^{\beta_{1}}\left(\frac{\alpha_{2}}{n}\right)^{\beta_{2}} \ldots\left(\frac{\alpha_{q}}{n}\right)^{\beta_{q}}, & \\
& \alpha_{1}+\alpha_{2}+\cdots+\alpha_{q}=n, \beta_{1}+\beta_{2}+\cdots+\beta_{q} .
\end{aligned}
$$

Barnett and Weaver considered the case $q=2$ [1].

K. J. Heuvers, et al, needed the fact that the matrix in (3) had an inverse in order to prove that the Cauchy-Binet theorem characterizes the permanent function [3]. This matrix has also been studied by R. Shelton, et al, while investigating multinomial Bernstein polynomials in [5].

Riordan obtained many of the results of this paper in the case $q=2$ when obtaining the inverses of certain Vandermonde matrices [4].

\section{Notation}

$$
\langle a\rangle_{0}=1, \quad\langle a\rangle_{n}=a(a-1) \cdots(a-n+1), \quad n \geq 1 .
$$

Let $\mathscr{F}$ be a field of characteristic $0, \alpha=\left(\alpha_{1}, \alpha_{2}, \ldots, \alpha_{q}\right) \in \mathscr{F}^{q}, q \geq 1$, $|\alpha|=\alpha_{1}+\alpha_{2}+\cdots+\alpha_{q}, \alpha^{\prime}=\left(\alpha_{1}, \alpha_{2}, \ldots, \alpha_{q-1}\right) \in \mathscr{F}^{q-1}, \alpha^{\prime \prime}=\left(\alpha_{1}, \alpha_{2}, \ldots\right.$, $\left.\alpha_{q-2}\right) \in \mathscr{F}^{q-2},\left|\alpha^{\prime}\right|=\sum_{i=1}^{q-1} \alpha_{i},\left|\alpha^{\prime \prime}\right|=\sum_{i=1}^{q-2} \alpha_{i}, \alpha=\left(\alpha^{\prime}, \alpha_{q}\right)$, and $|\alpha|=$ $\left|\alpha^{\prime}\right|+\alpha_{q}$. Also $x=\left(x_{1}, x_{2}, \ldots, x_{q}\right), x^{\prime}=\left(x_{1}, x_{2}, \ldots, x_{q-1}\right)$, and $x^{\prime \prime}=$ $\left(x_{1}, x_{2}, \ldots, x_{q-2}\right)$, where $x_{1}, x_{2}, \ldots, x_{q}$ are indeterminants.

$\mathbf{Z}_{+}=\{0,1,2, \ldots\} . P_{n, q}=\left\{\alpha \in \mathbf{Z}_{+}^{q}\right.$, with $\left.|\alpha|=n\right\} . I_{n, q}=\left\{\alpha^{\prime} \in \mathbf{Z}_{+}^{q-1}\right.$ with $\left.\left|\alpha^{\prime}\right| \leq n\right\}$. If $\alpha=\left(\alpha_{1}, \alpha_{2}, \ldots, \alpha_{q}\right) \in \mathbf{Z}_{+}^{q}$, let $x^{\alpha^{2}}=x_{1}^{\alpha_{1}} x_{2}^{\alpha_{2}} \cdots x_{q}^{\alpha_{q}}$ for $x=\left(x_{1}, x_{2}, \ldots, x_{q}\right)$. Similarly $x^{\prime \prime \alpha^{\prime}}=x_{1}^{\left(\alpha_{1}\right.} x_{2}^{\alpha_{2}} \cdots x_{q-1}^{\alpha_{q}-1},\left\langle x^{\prime}\right\rangle_{\alpha^{\prime}}=\left\langle x_{1}\right\rangle_{\alpha_{1}}\left\langle x_{2}\right\rangle_{\alpha_{2}} \ldots$ $\left\langle x_{q-1}\right\rangle_{\alpha_{q-1}}$ for $x^{\prime}=\left(x_{1}, x_{2}, \ldots, x_{q-1}\right)$ and $\alpha^{\prime} \in I_{n, q}$.

Also let $\alpha !=\alpha_{1} ! \alpha_{2} ! \cdots \alpha_{q} !, \alpha^{\prime} !=\alpha_{1} ! \alpha_{2} ! \cdots \alpha_{q-1} !$, and $\left(\begin{array}{c}\alpha^{\prime} \\ \beta^{\prime}\end{array}\right)=\left(\begin{array}{c}\alpha_{1} \\ \beta_{1}\end{array}\right)\left(\begin{array}{c}\alpha_{2} \\ \beta_{2}\end{array}\right) \cdots$ $\left(\begin{array}{c}\alpha_{q-1} \\ \beta_{q-1}\end{array}\right)=\left\langle\alpha^{\prime}\right\rangle_{\beta^{\prime}} / \beta^{\prime}$ !, for $\alpha^{\prime}, \beta^{\prime} \in I_{n, q}$. For $\alpha \in P_{n, q}$ the multinomial coefficient is $\left(\begin{array}{l}n \\ \alpha\end{array}\right)=n ! / \alpha$ ! and $|\alpha|=n$. There is a one-to-one correspondence between $\alpha^{\prime}=\left(\alpha_{1}, \alpha_{2}, \ldots, \alpha_{q-1}\right) \in I_{n, q}$ and $\alpha=\left(\alpha_{1}, \alpha_{2}, \ldots, \alpha_{q}\right) \in P_{n, q}$ given by $\alpha=\left(\alpha^{\prime}, n-\left|\alpha^{\prime}\right|\right)$. The equation

$$
\left(\begin{array}{l}
n \\
\alpha
\end{array}\right)=\frac{n !}{\alpha^{\prime} !\left(n-\left|\alpha^{\prime}\right|\right) !}=C\left(n, \alpha^{\prime}\right)
$$

defines the multinomial coefficient in terms of $\alpha^{\prime}$.

The ordering on $\mathbf{Z}_{+}^{q}$ is defined by $a<b$ if $a_{k}=b_{k}, k=1,2, \ldots, i-1$, and $a_{i}<b_{i}$. This is the lexicographic ordering. $a \ll b$ if $a_{i}<b_{i}$ for all $i$. The other symbols $\leq,>, \geq, \gg, \geq \geq, \leq \leq$ have obvious interpretations.

Let $\mathscr{F}_{n, q}=\left\{f \in \mathscr{F}\left[x_{1}, \ldots, x_{q-1}\right]: f=0\right.$ or $\left.\operatorname{deg} f \leq n\right\}$. A basis for $\mathscr{F}_{n, q}$ is given by $\left\{\left(x^{\prime}\right)^{r^{\prime}}: \alpha^{\prime} \in I_{n, q}\right\}$. 
Stirling numbers $S(k, m)$ of the second kind are defined by

$$
x^{m}=\sum_{k=0}^{m} S(k, m)\langle x\rangle_{k},
$$

and Stirling numbers $s(k, m)$ of the first kind are defined by

$$
\langle x\rangle_{m}=\sum_{k=0}^{m} s(k, m) x^{k} .
$$

Set $s(k, m)=S(k, m)=0$ whenever $k>m$.

The multinomial versions of these Stirling numbers can be defined by

$$
\mathscr{S}\left(\alpha^{\prime}, \beta^{\prime}\right)=\prod_{i=1}^{q-1} S\left(\alpha_{i}, \beta_{i}\right), \quad \alpha^{\prime}, \beta^{\prime} \in I_{n, q},
$$

and

$$
\mathbf{S}\left(\alpha^{\prime}, \beta^{\prime}\right)=\prod_{i=1}^{q-1} s\left(\alpha_{i}, \beta_{i}\right), \quad \alpha^{\prime}, \beta^{\prime} \in I_{n, q} .
$$

It should be pointed out that $\mathscr{S}\left(\alpha^{\prime}, \beta^{\prime}\right)=\mathbf{S}\left(\alpha^{\prime}, \beta^{\prime}\right)=0$ unless $\alpha^{\prime} \leq \leq \beta^{\prime}$.

- In addition $\mathscr{S}$ and $\mathbf{S}$ will denote matrices with elements $\mathscr{S}\left(\alpha^{\prime}, \beta^{\prime}\right)$ and $\mathbf{S}\left(\alpha^{\prime}, \beta^{\prime}\right)$ in the $\alpha^{\prime}, \beta^{\prime}$ position respectively, for $\alpha^{\prime}, \beta^{\prime} \in I_{n, q}$.

It will be assumed that the lexicographic ordering on $I_{n, q}$ will be used when forming the matrices and vectors indexed by $I_{n, q}$.

Note that the matrix $\left[\mathscr{S}\left(\alpha^{\prime}, \beta^{\prime}\right)\right]_{\alpha^{\prime}, \beta^{\prime} \in I_{n . q}}$ is upper triangular (when the lexicographic ordering of $I_{n, q}$ is used).

\section{The MUltinomial Dyson matrix}

Now the multinomial Dyson matrix will be defined by

$$
D_{n, q}=\left[\left(\begin{array}{c}
n \\
\beta
\end{array}\right)\left(\frac{\alpha}{n}\right)^{\beta}\right]_{\alpha, \beta \in P_{n, q}} .
$$

One other matrix that will play a significant role is given in the following theorem.

Theorem 1. Let $B$ be the matrix defined by

$$
B=\left[\left(\begin{array}{c}
\alpha^{\prime} \\
\beta^{\prime}
\end{array}\right)(-1)^{\left|\beta^{\prime}\right|}\right]_{\alpha^{\prime}, \beta^{\prime} \in I_{n \cdot 4}} .
$$

Then $B^{-1}=B$.

Proof. Let $X$ be the vector with components in $\mathscr{F}_{n, q}$ defined by

$$
X=\left[x^{\prime r^{\prime}}\right]_{r^{\prime} \in I_{n, q}} .
$$


The element of $B X$ with index $\alpha^{\prime}$ is

$$
\begin{aligned}
\sum_{\beta^{\prime} \in I_{n, q}}\left(\begin{array}{c}
\alpha^{\prime} \\
\beta^{\prime}
\end{array}\right)(-1)^{\left|\beta^{\prime}\right|} x^{\prime \beta^{\prime}} & =\sum_{\beta^{\prime} \in I_{n, q}} \prod_{i=1}^{q-1}\left(\begin{array}{c}
\alpha_{i} \\
\beta_{i}
\end{array}\right)\left(-x_{i}\right)^{\beta_{i}} \\
& =\prod_{i=1}^{q-1} \sum_{\beta_{i}=0}^{\alpha_{i}}\left(\begin{array}{c}
\alpha_{i} \\
\beta_{i}
\end{array}\right)\left(-x_{i}\right)^{\beta_{i}} \\
& =\prod_{i=1}^{q-1}\left(1-x_{i}\right)^{\alpha_{i}}
\end{aligned}
$$

This gives $B X=Y$ with $Y=\left[\prod_{i=1}^{q-1}\left(1-x_{i}\right)^{\alpha_{i}}\right]_{\alpha^{\prime} \in I_{n, q}}$. Now substitute $1-x_{i}$ for $x_{i}, i=1,2, \ldots, q-1$. This gives $B Y=X$. Hence $B^{2} X=X$ and since the elements of $X$ are linearly independent polynomials over the field $\mathscr{F}$, it must follow that $B^{2}=I$.

One consequence of this theorem is

Corollary 1. Let $C$ be the matrix defined by

$$
C=\left[\left(\begin{array}{c}
\alpha^{\prime} \\
\beta^{\prime}
\end{array}\right)\right]_{\alpha^{\prime}, \beta^{\prime} \in I_{n, q}}
$$

Then

$$
C^{-1}=\left[\left(\begin{array}{c}
\alpha^{\prime} \\
\beta^{\prime}
\end{array}\right)(-1)^{\left|\beta^{\prime}\right|-\left|\alpha^{\prime}\right|}\right]_{\alpha^{\prime}, \beta^{\prime} \in I_{n, q}} .
$$

Proof. Let $D$ be the diagonal matrix $\operatorname{diag}\left[(-1)^{\left|\alpha^{\prime}\right|}\right]_{\alpha^{\prime} \in I_{n, q}}$. Then $D^{-1}=D$ and $C=B D$. Then $C^{-1}=D B$ and that yields (13).

The main result now is:

Theorem 2. The eigenvalues of the Dyson matrix $D_{n, q}$ are given by

$$
\lambda_{\alpha^{\prime}}=\frac{n !}{n^{\left|\alpha^{\prime}\right|}\left(n-\left|\alpha^{\prime}\right|\right) !} \quad \text { where } \alpha^{\prime} \in I_{n, q}
$$

and there exists a complete set of linearly independent eigenvectors.

Proof. First a few lemmas are needed.

Lemma 1. The following equation holds.

$$
\sum_{\alpha^{\prime} \in I_{n, q}} C\left(n, \alpha^{\prime}\right)\left\langle\alpha^{\prime}\right\rangle_{\beta^{\prime}} x^{\prime \alpha^{\prime}}\left(n-\left|x^{\prime}\right|\right)^{n-\left|\alpha^{\prime}\right|}=\langle n\rangle_{\left|\beta^{\prime}\right|} x^{\prime \beta^{\prime}} n^{n-\left|\beta^{\prime}\right|} \text {. }
$$

Proof. Start with the multinomial theorem $|x|^{n}=\sum_{\alpha \in P_{n, q}}\left(\begin{array}{l}n \\ \alpha\end{array}\right) x^{\alpha}$, then apply the operators $\partial_{x_{1}}^{\beta_{1}} \partial_{x_{2}}^{\beta_{2}} \cdots \partial_{x_{q-1}}^{\beta_{q-1}}$. This gives

$$
\langle n\rangle_{\left|\beta^{\prime}\right|}|x|^{n-\left|\beta^{\prime}\right|}=\sum_{\alpha^{\prime} \in I_{n, q}} C\left(n, \alpha^{\prime}\right)\left\langle\alpha^{\prime}\right\rangle_{\beta^{\prime}} x^{\prime \alpha^{\prime}-\beta^{\prime}} x_{q}^{\alpha_{q}} .
$$


Next multiply both sides by $x^{\prime \beta^{\prime}}$, then substitute $n-\left|x^{\prime}\right|$ for $x_{q}$ in the resulting equation and the result follows.

The next lemma concerns Stirling numbers of the second kind.

Lemma 2. The following equation holds:

$$
\left(x^{\prime}\right)^{\beta^{\prime}}=\sum_{\gamma^{\prime} \in I_{n . q}} \mathscr{S}\left(\gamma^{\prime}, \beta^{\prime}\right)\left\langle x^{\prime}\right\rangle_{\gamma^{\prime}} .
$$

Moreover for all $\alpha^{\prime}, \beta^{\prime} \in I_{n, q}$ the following holds:

$$
\mathscr{S}\left(\alpha^{\prime}, \beta^{\prime}\right)=\frac{(-1)^{\left|\alpha^{\prime}\right|}}{\alpha^{\prime} !} \sum_{\gamma^{\prime} \in I_{n, q}}\left(\begin{array}{l}
\alpha^{\prime} \\
\gamma^{\prime}
\end{array}\right)(-1)^{\left|\gamma^{\prime}\right|} \gamma^{\prime \beta^{\prime}} .
$$

Proof. We start with

$$
\begin{aligned}
x^{\prime \prime \gamma^{\prime}} & =\prod_{i=1}^{q-1} x_{i}^{\left(\alpha^{i}\right.}=\prod_{i=1}^{q-1} \sum_{\gamma_{i}=0}^{\alpha_{i}} S\left(\gamma_{i}, \alpha_{i}\right)\left\langle x_{i}\right\rangle_{\gamma_{i}} \\
& =\sum_{\gamma^{\prime} \leq \leq \alpha^{\prime}} \prod_{i=1}^{q-1} S\left(\gamma_{i}, \alpha_{i}\right)\left\langle x_{i}\right\rangle_{\gamma_{i}}=\sum_{\gamma^{\prime} \leq \leq \alpha^{\prime}} \mathscr{S}\left(\gamma^{\prime}, \alpha^{\prime}\right)\langle x\rangle_{\gamma^{\prime}}=\sum_{\gamma^{\prime} \in I_{n . q}} S\left(\gamma^{\prime}, \alpha^{\prime}\right)\langle x\rangle_{\gamma^{\prime}} .
\end{aligned}
$$

One of the standard closed form expressions for Stirling numbers of the second kind is given by [2, p. 119]

$$
S(m, n)=\frac{(-1)^{m}}{m !} \sum_{k=0}^{m}\left(\begin{array}{c}
m \\
k
\end{array}\right)(-1)^{k} k^{n} .
$$

This implies that

$$
\begin{aligned}
\mathscr{S}\left(\alpha^{\prime}, \beta^{\prime}\right) & =\prod_{i=1}^{q-1} S\left(\alpha_{i}, \beta_{i}\right) \\
& =\prod_{i=1}^{q-1} \frac{(-1)^{\alpha_{i}}}{\alpha_{i} !} \sum_{\gamma_{i}=0}^{\alpha_{i}}\left(\begin{array}{c}
\alpha_{i} \\
\gamma_{i}
\end{array}\right)(-1)^{\eta^{\prime}} \gamma_{i}^{\beta_{i}} \\
& =\frac{(-1)^{\left|\alpha^{\prime}\right|}}{\alpha^{\prime} !} \sum_{\gamma^{\prime} \leq \leq \alpha^{\prime}} \prod_{i=1}^{q-1}\left(\begin{array}{c}
\alpha_{i} \\
\gamma_{i}
\end{array}\right)(-1)^{\gamma^{\prime}} \gamma_{i}^{\beta_{i}} \\
& =\frac{(-1)^{\left|\alpha^{\prime}\right|}}{\alpha^{\prime} !} \sum_{\gamma^{\prime} \leq \leq \alpha^{\prime}}\left(\begin{array}{c}
\alpha^{\prime} \\
\gamma^{\prime}
\end{array}\right)(-1)^{\left|\gamma^{\prime}\right|} \gamma^{\prime \beta^{\prime}} .
\end{aligned}
$$

The lemma now follows. 
First compute the matrix $D B$ whose $\alpha^{\prime}, \beta^{\prime}$ element is

$$
\begin{aligned}
\sum_{\gamma^{\prime} \in I_{n . q}} D_{\left(\alpha^{\prime}, \gamma^{\prime}\right.} B_{\gamma^{\prime}, \beta^{\prime}} & =\sum_{\gamma^{\prime} \in I_{n, q}} C\left(n, \gamma^{\prime}\right)\left(\frac{\alpha^{\prime}}{n}\right)^{\gamma^{\prime}}\left(1-\frac{\left|\alpha^{\prime}\right|}{n}\right)^{n-\left|\gamma^{\prime}\right|}\left(\begin{array}{c}
\gamma^{\prime} \\
\beta^{\prime}
\end{array}\right)(-1)^{\left|\beta^{\prime}\right|} \\
& =\frac{(-1)^{\left|\beta^{\prime}\right|}}{n^{n} \beta^{\prime} !} \sum_{\gamma^{\prime} \in I_{n, q}} C\left(n, \gamma^{\prime}\right) \alpha^{\prime \gamma^{\prime}}\left(n-\left|\alpha^{\prime}\right|\right)^{n-\left|\gamma^{\prime}\right|}\left\langle\gamma^{\prime}\right\rangle_{\beta^{\prime}} .
\end{aligned}
$$

Now use Lemma 1 to get

$$
D B=\left[\frac{(-1)^{\left|\beta^{\prime}\right|}\langle n\rangle_{\left|\beta^{\prime}\right|} \alpha^{\prime \beta^{\prime}}}{n^{\left|\beta^{\prime}\right|} \beta^{\prime} !}\right]_{\alpha^{\prime}, \beta^{\prime} \in I_{n . q}} .
$$

Next we compute $B D B$. The $\alpha^{\prime}, \beta^{\prime}$ element of $B D B$ is

$$
\frac{(-1)^{\left|\beta^{\prime}\right|}\langle n\rangle_{\left|\beta^{\prime}\right|}}{n^{\left|\beta^{\prime}\right|} \beta^{\prime} !} \sum_{\gamma^{\prime} \in I_{n, q}}\left(\begin{array}{l}
\alpha^{\prime} \\
\gamma^{\prime}
\end{array}\right)(-1)^{\gamma^{\prime}} \gamma^{\prime \beta^{\prime}} \text {. }
$$

Now use Lemma 2 on the sum to get

$$
\frac{\alpha^{\prime} !(-1)^{\left|\beta^{\prime}\right|-\left|\alpha^{\prime}\right|}\langle n\rangle_{\left|\beta^{\prime}\right|}}{\beta^{\prime} ! n^{\left|\beta^{\prime}\right|}} \mathscr{S}\left(\alpha^{\prime}, \beta^{\prime}\right) .
$$

This yields the important equation

$$
T=B D B=\left[\frac{\alpha^{\prime} !(-1)^{\left|\beta^{\prime}\right|-\left|\alpha^{\prime}\right|}\langle n\rangle_{\left|\beta^{\prime}\right|}}{\beta^{\prime} ! n^{\left|\beta^{\prime}\right|}} \mathscr{S}\left(\alpha^{\prime}, \beta^{\prime}\right)\right]_{\alpha^{\prime}, \beta^{\prime} \in I_{n, q}} .
$$

It now follows that $D$ is similar to an upper triangular matrix $T$ whose diagonal entries are $\lambda_{\beta^{\prime}}=\langle n\rangle_{\left|\beta^{\prime}\right|} / n^{\left|\beta^{\prime}\right|}$. Therefore these are the eigenvalues of $D$.

Next, it is necessary to show that there exists a complete linearly independent set of eigenvectors corresponding to each eigenvalue $\lambda_{b}=\langle n\rangle_{b} / n^{b}$. Notice that $1=\lambda_{0}=\lambda_{1}>\lambda_{2}>\lambda_{3}>\cdots>\lambda_{n}$. Let $S_{b}=\left\{\alpha^{\prime} \in I_{n, q}:\left|\alpha^{\prime}\right|=b\right\}$ for $b>1$, and $S_{1}=\left\{\alpha^{\prime} \in I_{n, q}:\left|\alpha^{\prime}\right|=0\right.$ or $\left.\left|\alpha^{\prime}\right|=1\right\}$. If $\left|\alpha^{\prime}\right|=\left|\beta^{\prime}\right|$ and $\alpha^{\prime} \leq \leq \beta^{\prime}$ then $\alpha^{\prime}=\beta^{\prime}$. Therefore $\left|\alpha^{\prime}\right|=\left|\beta^{\prime}\right|$ and $\alpha^{\prime} \neq \beta^{\prime}$ implies that $\mathscr{S}\left(\alpha^{\prime}, \beta^{\prime}\right)=T_{r^{\prime}, \beta^{\prime}}=0$. If $\left|\alpha^{\prime}\right|=0$ and $\left|\beta^{\prime}\right|=1$, then $\alpha^{\prime}=0$, and $\beta^{\prime}$ has one component equal to one and the rest of the components are zero. From (15) $\mathscr{S}\left(\alpha^{\prime}, \beta^{\prime}\right)=0$. Hence if $\alpha^{\prime}$ and $\beta^{\prime}$ are in $S_{b}$ and $\alpha^{\prime} \neq \beta^{\prime}$ then $T_{\alpha^{\prime}, \beta^{\prime}}=0$.

Let $T_{b}$ be the submatrix of $T$ consisting of elements with both indices in $S_{b}$. Then $T_{b}=\lambda_{b} I$ has a complete set of linearly independent eigenvectors corresponding to $\lambda_{b}$. The diagonal elements of $T$ not belonging to $T_{b}$ are not equal to $\lambda_{b}$. Hence, all the eigenvectors of $T_{b}$ can be extended to linearly independent eigenvectors of $T$. 
The argument is true for all the eigenvalues of $T$, thus $T$ has a complete set of linearly independent eigenvectors. The same is true for $D$ since $T$ is similar to $D$ by $(20)$.

This result has a number of consequences, one of them is that the Dyson matrix is invertible, since all the eigenvalues are nonzero. In fact the inverse Dyson matrix can be expressed in terms of multinomial Stirling numbers of the first kind and that is what will be done next.

Just as Stirling numbers of the second kind have a multinomial extension, so do Stirling numbers of the first kind. In fact the following counterpart of Lemma 2 can be established.

Lemma 3. The following equation holds:

$$
\left\langle x^{\prime}\right\rangle_{\alpha^{\prime}}=\sum_{\beta^{\prime} \in I_{n . q}} \mathbf{S}\left(\beta^{\prime}, \alpha^{\prime}\right) x^{\prime \beta^{\prime}} .
$$

Moreover the matrix $\mathbf{S}$ is the inverse of the matrix $\mathscr{S}$. Proof.

$$
\begin{aligned}
\left\langle x^{\prime}\right\rangle_{\alpha^{\prime}} & =\prod_{i=1}^{q-1}\langle x\rangle_{\alpha^{\prime}}=\prod_{i=1}^{q-1} \sum_{\beta_{i}=0}^{\alpha_{i}} s\left(\beta_{i}, \alpha_{i}\right) x_{i}^{\beta_{i}}=\sum_{\beta^{\prime} \leq \leq \alpha^{\prime}}\left(\prod_{i=1}^{q-1} s\left(\beta_{i}, \alpha_{i}\right) x_{i}^{\beta_{i}}\right) \\
& =\sum_{\beta^{\prime} \leq \leq \alpha^{\prime}} \mathbf{S}\left(\beta^{\prime}, \alpha^{\prime}\right) x^{\beta^{\prime}}=\sum_{\beta^{\prime} \in I_{n . q}} \mathbf{S}\left(\beta^{\prime}, \alpha^{\prime}\right) x^{\prime \beta^{\prime}} .
\end{aligned}
$$

(21) and (15) imply that $\mathscr{S}$ is the change of basis matrix from the basis $\left\{x^{\prime r^{\prime}}\right\}_{\alpha^{\prime} \in I_{n, q}}$ of $\mathscr{F}_{n, q}$ to the basis $\left\{\left\langle x^{\prime}\right\rangle_{\alpha^{\prime}}\right\}_{\alpha^{\prime} \in I_{n, q}}$, and $\mathbf{S}$ is the change of basis matrix from the basis $\left\{\left\langle x^{\prime}\right\rangle_{\alpha^{\prime}}\right\}_{\alpha^{\prime} \in I_{n, q}}$ to the basis $\left\{x^{\prime \alpha^{\prime}}\right\}_{\alpha^{\prime} \in I_{n, q}}$. Thus $\mathscr{S}$ and $\mathbf{S}$ are inverses of each other. This proves the lemma.

Now define two diagonal matrices as follows

$$
L=\operatorname{diag}\left[\frac{(-1)^{\left|\alpha^{\prime}\right|}}{\alpha^{\prime} !}\right]_{\alpha^{\prime} \in I_{n . q}},
$$

and

$$
\Lambda=\operatorname{diag}\left[\frac{\langle n\rangle_{\left|\alpha^{\prime}\right|}}{\alpha^{\prime} !}\right]_{\alpha^{\prime} \in I_{n, q}} .
$$

These observations can be summarized in the following corollary.

Corollary 2. With $L$ and $\Lambda$ defined in (22) and (23) above, the following holds:

$$
D=B L^{-1} \mathscr{S} \Lambda L B
$$

and

$$
D^{-1}=B L^{-1} \Lambda^{-1} \mathbf{S} L B
$$




\section{REFERENCES}

1. F. C. Barnett and J. R. Weaver, Eigenvalues and eigenvectors of a certain stochastic matrix, Linear and Multilinear Algebra 13 (1983), 345-350.

2. D. A. Cohen, Basic techniques of combinatorial theory, John Wiley and Sons, Inc., New York, 1978.

3. K. J. Heuvers, L. J. Cummings and K. P. S. Bhaskara Rao, A characterization of the permanent function by the Binet-Cauchy Theorem, Linear Algebra Appl. 101 (1988), 49-72.

4. J. Riordan, Combinatorial identities, John Wiley and Sons, Inc., New York, 1968.

5. R. Shelton, K. J. Heuvers, K. P. S. Bhaskara Rao and D. S. Moak, Multinomial matrices, Discrete Math. 61 (1986), 107-114.

Department of Mathematics, Michigan Technological University, houghton, MiCHIGAN 49931 